\title{
Review on Memory Recall via Image Processing Intelligent Using Social Contacts Based on Eigen Value Implementation
}

\author{
Miss. Snehal S. Gulhane ${ }^{1}$, Prof. A. S. Kapse ${ }^{2}$ \\ M.E. Student, CSE, PRPCET, Amravati, Maharashtra, India ${ }^{1}$ \\ Assistant Professor, CSE, PRPCET, Amravati, Maharashtra, India ${ }^{2}$
}

\begin{abstract}
Human memory very often fails. People are frequently beset with questions like "Who is that person? I think I met him/her last year." Available memory systems cannot well support the recall of names effectively. This paper explores the memory recall enhancement problem from the perspective of memory cue extraction and associative search, and proposes a generic method to extract memory cues from heterogeneous, multimodal, physical/virtual data sources. Specifically, we can use the contact name recall in the academic community as the target application to showcase our proposed methodology. We next develop an intelligent social contact manager that supports 1) auto collection of rich contact data from a combination of various pervasive sensors and Web data sources, and 2) associative search of contacts when human memory fails. The proposed system is validated with the help of testing the performance of contact data collection techniques. An user study on contact memory recall is conducted, through which several findings about contact memorizing and recall are presented. Various Classic cognitive psychology theories are used to interpret these findings [1].
\end{abstract}

Keywords: Contact recall, memory aid, pervasive computing, social contact management, Web intelligenc.

\section{INTRODUCTION}

Memory lapse is a common problem among people. Many different forms of memory lapses occur in our daily life. For e.g., we may forget the name of a contact person or the title of a document. These memory failures bring many inconveniences to our work and life. Hence, finding effective ways to aid human memory becomes the fundamental and challenging problem. Enhancement to memory recall has been investigated in diverse areas for a long time. Before the time of computing, it was done manually. Representative examples are always note taking and address-book writing. However, these methods suffer from problems, including possible loss or damage and inefficient search.

In today's life, people participate in various social activities and meet numerous people every day. These acquaintances form the social contact network (SCN) of a person. Thus, the ability to manage the SCN and use it to get things done has become a difficult task. For example, Bob is an active researcher. He participates in various academic activities and meets numerous new contacts. To maintain his SCN efficiently, Bob uses a digital contact book for storing the information of his contacts. However, he encounters two issues. First, contact information retrieval can be difficult. Finding the needed information is not difficult using a traditional contact tool if we know the name of the target contact. Just like most people, however, Bob often forgets the names of his contacts. For example, while at the airport, Bob sees someone he has met before. He wants to talk to that person but cannot recall the information not even the person's name. The following is what he could only recall: "I met him at a conference in Tokyo in 2009. He told me that he graduated from MIT, with Web mining as his research interest." As an academic community member, Bob pays particular attention to some academic information of a contact, such as the contact's educational background and research interest. The contextual information relevant to the meeting event with the contact is also easy to remember. However, traditional contact tools do not work with such memory cues. Second, the cost of manually collecting the aforementioned memory cues is quite high. Thus, it is better to develop a tool that can automatically collect the needed contact information

\section{Objective}

1. The face recognisation will be implemented using advance techniques like eigen value determination

2. Determination the facial parameters and used them for efficient face recognition technique

3. Implementation of fast auto retrieval of dataset from social media datasource.

4. Evolutionary determination of efficient memory recall with minimum timeconsumption.

5. Implementation of value matching techniques for exact value determination

\section{LITERATURE REVIEW}

Use of memory cues and the improvements in social contact management systems for the enhancement of memory recall has brought closer some related search directions [2]: 


\section{A. Digital Memory Aids}

Digital memory aid systems basically fell in to two categories depending upon the targeted memory issues. The issues are, Retrospective aid, and prospective aid [3].Retrospective aid basically works with the recall of the past events, previously held events; whereas, the Prospective aid deals with remembering the future events. We have concentrated our study on the retrospective aid, rather than prospective aid. Retrospective memory aid has two important application domains:

\section{B. Digital Object Re-finding}

The main focus of the Digital object refinding is on the storage and search of collections of heterogeneous digital objects. These objects are either generated by the users or been encountered at the time of work processes, like emails, documents etc [4],[5],[6] . Metadata on the diverse aspects of an object's context like, when, where and relationships amongst them, is used commonly by tools. This makes the re-finding of the information much easier.

\section{Life-Logging}

Unlike the digital object re-finding, the Life-Logging extends beyond the storage of objects in closed environment, rather it moves in the real world. Because of this, it can capture our common activities. For Life logging, various systems have been developed. These systems commonly use the portable and sometimes wearable devices for capturing the data. A personal digital assistant [PDA] [7] is used by the Forget-me-not. Because of this, it can capture the activities of its users in the text form, i.e. user locations etc, daily.

\subsection{Human-Centric Computing}

The context-aware services are provided by the Humancentric computing with respect to the extracted information from the cyber-physical space about the user. There are two major sources for extracting the human information: web and pervasive sensors. Both these sources acquire the different attributes and strengths. The web is the best source for the static or slowly changing human information. This information may include the user profiles, social relationships etc. whereas; the pervasive sensing allows detecting the human activities, their social interactions, etc within the existent world. Combined data from these distinct sources allows exclusive opportunities to pervasive applications because of the diverse features. But somehow, till the date, both these sources have followed the different research fields. Few systems have been developed in order to combine the power of these two data sources additionally. We have proposed a unique method to auto collect the digital traces of humans from the heterogeneous datasets.

\section{Social Contact Tools}

Assisting the communication with contacts and the recommended contacts, many systems have been developed.

\section{Contact communication Environments}

Suiting electronic communications like, emails, instant messaging or social networking sites have become increasingly reliable for the modern work. An alternative representation of the emails is provided by the Contact Map .A problem that occurs regularly with such systems is that, they are strictly created for the communications between registered users in the virtual world. A general way of allowing people to create digital connections with their contacts is proposed by the Social Contact Manager (SCM) [8]. Exchanging the business cards in real world is the only source for connections

\section{Contact recommendation}

Many systems have been developed to recommend the users with the new contacts. The recommendations are based on the background, social relationships, or context of the users. The users inside a public place, like hotels, can exchange the social network IDs by using their mobile phones and find someone of their interest. The who that provided this unique feature. Our system tends to support the management and already acquired information's recollection, rather than giving a social matching service. For getting the work done, personal contacts are critical.

\subsection{Eigen Face Process}

There are two main jobs in the face recognition system. The jobs are enrollment and identification. In the enrollment process there are the stages in the eigenface calculation, i.e. image acquisition, preprocessing and the threshold value for each image. While in the identification process stages are image acquisition, preprocessing and recognize the test image with Eigen face process [9].

\section{PROPOSED WORK}

1. In existing work the keyword base searching pattern is used which is not too much efficient for searching.

2. No image based approach is used.

3.1 Need \& Significance of proposed system

1. Proposed system will work on image identification

2. Accuracy of determination exact title increases.

3. The image determination implemented using EIGENFACE METHOD in android.

4. In proposed work the time lapse

5. Feasible excess to Social Contact Network

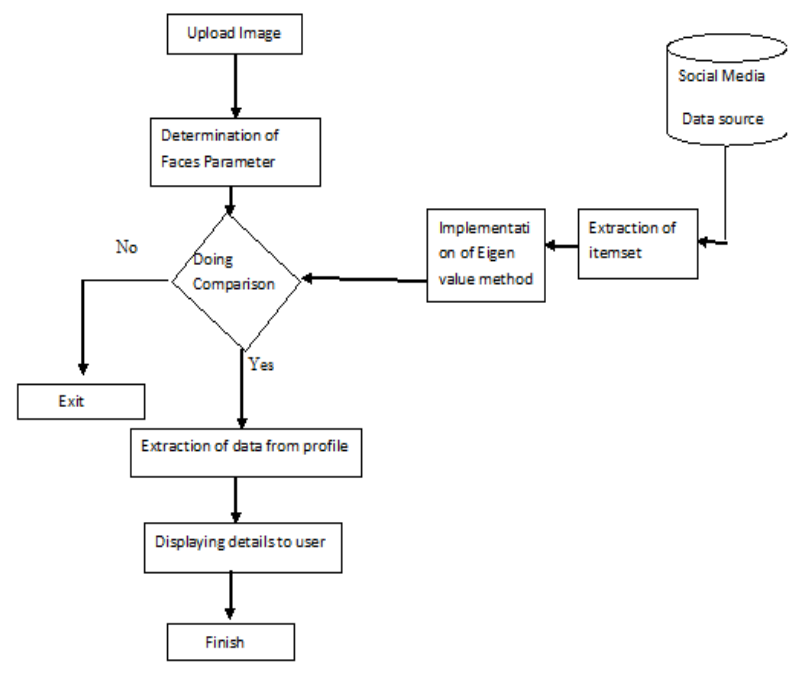




\subsection{Methodology}

1. Capturing Image using android face detection

2. Extraction of profiles from Facebook friend list using Facebook API

3. Determination of Eyes distance using Eighen value implementation

4. comparing values with extracted data set

5. calculating matching ration

6. Perception of image

\section{CONCLUSION}

Memory lapse is big problem in humans to reduce this problem and proposed the effective image processing in concerning with social media data source is big challenge. the vast processing within the millions of record, reducing the time estimation and increasing accuracy will provide the new functionality to recall for society. develop a system which helps the people to recall through social contact network \& image processing with eigen face method of android. the proposed system will helps to reduce the time consumption for accessing the $\mathrm{scn}$.

\section{REFERENCES}

[1] Bin Guo, Member, IEEE, Daqing Zhang, Member, IEEE, Dingqi Yang, Zhiwen Yu, Senior Member, IEEE , and Xing she Zhou, Member, IEEE "Enhancing Memory Recall via an IntelligentSocial Contact Management System" VOL. 44, NO. 1, FEBRUARY 2014.

[2] Nitin Ingle1 Prof. Ayesha Butalia2 'Social Contact Management System for Memory Recall Enhancement 'International Journal of Innovations \& Advancement in Computer Science IJIACS ISSN 2347 - 8616 Volume 3, Issue 9 November 2014.

[3] D. Elsweiler, I. Ruthven, and C. Jones,"Towards memory supporting personal information management tools," J. Amer. Soc. Inform. Sci. Technol., 2007.

[4] D. Elsweiler, M. Baillie, and I. Ruthven,"Exploring memory in email refinding," ACM Trans. Inf. Syst., 2008.

[5] D. H. Chau, B. Myers, and A. Faulring,"What to do when search fails: Finding information by association," in Proc. Conf. Human Factors Comput . Syst., 2008.

[6] D. Zhang, B. Guo, and Z. Yu, "The emergence of social and community intelligence," IEEE Comput., 2011.

[7] M. Lamming and M. Flynn, "Forgetme-not: Intimate computing in support of human memory," in Proc. FRIEND21,1994.

[8] Shailesh U. Sambhe, SachinMurab Literature Review on Smart Social Contact Management System for Better Memory Recall ISO 9001:2008 Certified International Journal of Engineering and Innovative Technology (IJEIT) Volume 3, Issue 9, March 2014.

[9] Oka Sudana, a. a. k., Darma Putra, i k. g., Aalanarismandika face recognition system on android using eigen face method Journal of Theoretical and Applied Information Technology10th March 2014. Vol. 61 No.1. 